University of Wollongong

Research Online

Australian Institute for Innovative Materials -

Papers

Australian Institute for Innovative Materials

$1-1-2014$

One-pot aqueous synthesis of cysteine-capped $\mathrm{CdTe} / \mathrm{CdS}$ core-shell nanowires

Yukai Shan

Yunnan University

Zhen Xiao

Yunnan University

Yongming Chuan

Yunnan University

Hongli Li

Yunnan University

Minglong Yuan

Yunnan University

See next page for additional authors

Follow this and additional works at: https://ro.uow.edu.au/aiimpapers

Part of the Engineering Commons, and the Physical Sciences and Mathematics Commons

Research Online is the open access institutional repository for the University of Wollongong. For further information contact the UOW Library: research-pubs@uow.edu.au 


\title{
One-pot aqueous synthesis of cysteine-capped CdTe/CdS core-shell nanowires
}

\begin{abstract}
Highly fluorescent cysteine-capped $\mathrm{CdTe} / \mathrm{CdS}$ core-shell nanowires were successfully synthesized by reacting $\mathrm{CdCl} 2$ with $\mathrm{NaHTe}$ in aqueous solution under refluxing at $100{ }^{\circ} \mathrm{C}$ for $140 \mathrm{~min}$. On increasing the reaction time from 10 to $140 \mathrm{~min}, \mathrm{CdTe} / \mathrm{CdS}$ nanocrystals gradually grew into nanorods and eventually completely evolved into nanowires. The nanowires have amino and carboxyl functional groups on their surfaces and can be well dispersed in aqueous solution. The as-prepared CdTe/CdS nanowires show a fluorescence quantum yield (QY) of $7.25 \%$ due to the unique nature of cysteine and the formation of a CdS shell on the surface of the CdTe core, they have a narrower diameter distribution $(\mathrm{d}=\sim 5 \mathrm{~nm})$ and a length in the range of $175-275 \mathrm{~nm}$, and their aspect ratio is between $1 / 35$ and 1/55.
\end{abstract}

\section{Keywords}

cds, core, shell, nanowires, one, pot, aqueous, synthesis, cysteine, capped, cdte

Disciplines

Engineering | Physical Sciences and Mathematics

\section{Publication Details}

Shan, Y., Xiao, Z., Chuan, Y., Li, H., Yuan, M., Li, Z. \& Dou, S. (2014). One-pot aqueous synthesis of cysteinecapped CdTe/CdS core-shell nanowires. Journal of Nanoparticle Research, 16 (2420), 1-11.

\section{Authors}

Yukai Shan, Zhen Xiao, Yongming Chuan, Hongli Li, Minglong Yuan, Zhen Li, and S X. Dou 


\section{One-pot Aqueous Synthesis of Cysteine-Capped CdTe/CdS Core-shell Nanowires}

Yukai Shan ${ }^{\mathrm{a}}$, Zhen Xiao ${ }^{\mathrm{a}}$, Yongming Chuan ${ }^{\mathrm{a}}$, Hongli Li ${ }^{\mathrm{a}}$, Minglong Yuan ${ }^{\mathrm{a} *}$, Zhen

$\mathrm{Li}^{\mathrm{b} *}$, and Shixue Dou ${ }^{\mathrm{b}}$

${ }^{a}$ Engineering Research Center of Biopolymer Functional Materials of Yunnan,

Yunnan University of Nationalities, Kunming 650500, China

${ }^{\mathrm{b}}$ Institute for Superconducting \& Electronic Materials, University of Wollongong, Innovation Campus, Squires Way, North Wollongong, NSW 2500, Australia

* Corresponding author: Minglong Yuan. Tel.: +86-18987188989.

E-mail address: yml@188.com; Zhen Li: Email: zhenl@uow.edu.au. 


\section{ABSTRACT}

Highly fluorescent cysteine-capped CdTe/CdS core-shell nanowires were successfully synthesized by reacting $\mathrm{CdCl}_{2}$ with $\mathrm{NaHTe}$ in aqueous solution under refluxing at $100{ }^{\circ} \mathrm{C}$ for $140 \mathrm{~min}$. On increasing the reaction time from 10 to $140 \mathrm{~min}$, CdTe/CdS nanocrystals gradually grew into nanorods and eventually completely evolved into nanowires. The nanowires have amino and carboxyl functional groups on their surfaces and can be well dispersed in aqueous solution. The as-prepared CdTe/CdS nanowires show a fluorescence quantum yield (QY) of $7.25 \%$ due to the unique nature of cysteine and the formation of a CdS shell on the surface of the CdTe core, they have a narrower diameter distribution $(d=\sim 5 \mathrm{~nm})$ and a length in the range of 175 to $275 \mathrm{~nm}$, and their aspect ratio is between 1/35 and 1/55.

KEYWORDS. CdTe/CdS, core-shell nanowires, aqueous preparation, cysteine.

\section{INTRODUCTION}

In recent years, one-dimensional (1D) semiconductor nanostructures such as nanorods, nanowires, and nanotubes have attracted considerable attention because of their special structures, unique electrical and optical properties, and diverse applications (Tang et al. 2002; Yong et al. 2010). Due to their unique physical and chemical properties with high dimensional anisotropy, semiconductor nanowires are of particular importance for fabricating nanoscale biosensors (Fernando et al. 2007; 
Jay et al. 2009), drug carriers (Lee et al. 2009), solar cells (Jiang et al. 2010; Yu et al. 2010), and cell imaging devices (Hwa et al. 2009). For example, II - VI CdTe nanowires (Luo et al. 2012) have an extremely high absorption coefficient, broad absorption range, and excellent carrier transport properties, so that they are ideal candidates for the above-mentioned applications (Jiang et al. 2012).

Different techniques have been developed to synthesize nanowires, which can be generally classified into top-down and bottom-up routes. A conventional bottom-up method is vapor-liquid-solid (VLS) growth (Simon et al. 2010; Wu et al. 2012; Wang et al. 2008; Yang et al. 2013), in which nanowire diameters are controlled by the size of the Au or Bi nanocatalysts. The obtained nanowires have diameters of about 20-60 $\mathrm{nm}$, and are usually not within the quantum confinement region. They also have poor hydrophilicity, and the preparation process requires high temperature, usually higher than $500{ }^{\circ} \mathrm{C}$. In addition,the nanocatalysts that need to be used remain at the ends of the nanowires, which could influence nanowire properties and performance. Another common approach is the chemical vapor deposition (CVD) method (Hou et al. 2011; Park et al. 2008; Carey et al. 2009; Utama et al. 2011), which can produce nanowires several micrometers in length at high temperature (more than $500{ }^{\circ} \mathrm{C}$ ). Similar to VLS growth, the prepared nanowires are usually larger than $50 \mathrm{~nm}$ in diameter.

Compared with above mentioned non-wet chemical methods, preparation of nanowires by wet-chemical approaches has a few distinct advantages. First, the resultant semiconductor nanowires have smaller diameters and a narrower diameter 
distribution, and they can exhibit quantum confinement effects like their nanocrystal and nanorod analogues. Secondly, the resultant colloidal nanowires can be further functionalized by post-surface modification. Thirdly, the reaction temperature is lower. For these reasons, we used Bi nanoparticles as catalysts to prepare colloidal semiconductor nanowires through solution-liquid-solid (SLS) growth at relatively low temperature (300 ${ }^{\circ} \mathrm{C}$ ) ( $\mathrm{Li}$ et al. 2010; Wang et al. 2011; Li et al. 2012; Li et al. 2011; Anton et al. 2011; Li et al. 2008; Li et al. 2009). The diameter of these colloidal nanowires can be tuned from a few nanometers to tens of nanometers, so that they exhibit strong quantum confinement effects. Their optical, electronic, and magnetic properties can be altered by in-situ chemical doping or post surface modification. For example, the photostability of CdSe nanowires can be improved by growing a shell on their surfaces to from core-shell structures, which have significantly improved fluorescence quantum yields (QYs) to 2\%, 100 times higher than that of the original CdSe core (Goebl et al. 2008).

This versatile SLS growth is capable of producing colloidal semiconductor nanowires from groups IV (Si, Ge), II-VI (CdS, CdSe, CdTe), III-V (InP, GaP), and IV-VI (PbS, $\mathrm{PbSe}, \mathrm{PbTe}$ ). These nanowires are hydrophobic, however, and have low fluorescence quantum yield, which limits their bioapplications. In addition, SLS growth requires an air-free environment due to the use of air-sensitive chemicals. Such air-free manipulations significantly increase the complexity as well as the cost of the synthesis 
of colloidal nanowires. Therefore, it is highly desirable to prepare high quality water-soluble fluorescent nanowires for bioapplications.

Compared with vapor-phase and organic-phase syntheses, aqueous preparations are simpler, cheaper, and more environmentally friendly. More importantly, the obtained nanowires are naturally water-dispersed without any post-treatment due to the presence of a large amount of hydrophilic ligands (Chen et al. 2012).There are some reports on the preparation of CdTe nanowires in aqueous phase (Liang et al. 2009; Yong et al. 2011; Deng et al. 2013; Bao et al. 2006; Niu et al. 2005; Jiang et al. 2013). A big issue for these nanowires, however, is the presence of many defects on their surfaces, which will significantly degrade their optical, electrical, and thermodynamic performance (Jiang et al. 2012). In order to reduce the surface defects, the core-shell structure has been introduced and has proven to be a powerful approach to improve the overall physical and chemical properties of the nanowires. Core-shell CdTe/CdS nanowires are a type-I structure, in which both the valence and conduction band edges are respectively lower and higher than those of the core. Such core-shell nanowires exhibit higher fluorescence quantum yield than core nanowires (Gui et al. 2013). More importantly, the core-shell structure can effectively reduce the toxicity of the nanowires. Thus, such heterostructured nanowires show great potential for a new generation of biosensors and have attracted strong interest from both the scientific and the industrial community. There are only a few reports, however, on preparation of functional CdTe/CdS core-shell nanowires in aqueous phase, which may be due to the 
large lattice mismatch between CdTe and CdS, as well as additional difficulties associated with the morphological anisotropy of CdTe core nanowires (Wang et al. 2008).

Here, we report an environmentally friendly one-pot aqueous reaction to prepare CdTe/CdS core-shell nanowires functionalized with cysteine, cysteine as a small biomolecule, has been introduced to controllably synthesize various metal sulfides in hydrothermal/solvothermal routes, because the sulfhydryl group of cysteine is generally the most reactive functional group in a protein (Laura et al. 2003). The resultant core-shell nanowires show a quantum yield of 7.5\%, low cytotoxicity, and good potential for cell labeling.

\section{Experimental}

\subsection{Materials}

Cadmium chloride anhydrous $\left(\mathrm{CdCl}_{2}\right)$, tri-sodium citrate dehydrate (99.3\%), sodium borohydride ( $\left.\mathrm{NaBH}_{4}, 99 \%\right)$, L-cysteine (Cys), and Te powder (99.9\%) were used as received without further purification. Milli-Q water (>18 M $\Omega$ ) was used in all experiments.

\subsection{Preparation of NaHTe Solution}

$5.1 \mathrm{mg}$ Te powder and $61 \mathrm{mg}$ sodium borohydride $\left(\mathrm{NaBH}_{4}\right)$ were transferred into a $10 \mathrm{~mL}$ flask, then $2 \mathrm{~mL}$ of ultrapure water was added, and the mixture was stirred at 
room temperature. During the reaction, a small outlet connected to the flask was kept open to discharge the pressure from the resulting hydrogen. The reaction was finished when the solid was completely dissolved, resulting in a clear solution.

\subsection{Preparation of $\mathrm{CdTe} / \mathrm{CdS}$ nanowires}

Cadmium chloride solution $\left(\mathrm{CdCl}_{2}, 0.04 \mathrm{M}, 4 \mathrm{~mL}\right)$ was first diluted to $50 \mathrm{~mL}$ in a one-necked flask, and then trisodium citrate dehydrate (400 mg), NaHTe solution (2 $\mathrm{mL}$ ), and cysteine (Cys, $82 \mathrm{mg}$ ) were added under vigorous stirring. After the solution became orange, the flask was attached to a condenser and refluxed at $100{ }^{\circ} \mathrm{C}$ for the desired period of time (10-180 min) under Ar flow. The solution was then cooled down to room temperature, and excess ethanol was added to the nanowire aqueous solution. The precipitated nanowires were collected via centrifugation (8000 rpm, 5 min). After decanting of the supernatant, the nanowires were redispersed in Milli-Q water and reprecipitated by ethanol. The same procedure was repeated 3 times to thoroughly wash away free ions. The nanowires were finally dispersed in Milli-Q water for further characterization (yeild: 21.7\%). The CdTe/CdS nanowires were synthesized according to the following chemical reaction:

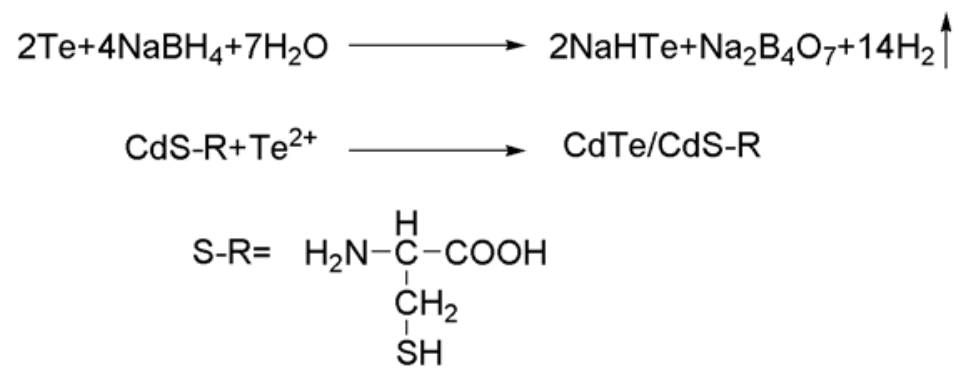


During the reaction, $\mathrm{NaBH}_{4}$ reduces $\mathrm{Te}$ to $\mathrm{Te}^{2+}$, the fresh $\mathrm{Te}^{2-}$ reacts with $\mathrm{Cd}^{2+}$ to give CdTe core, and cysteine combine with $\mathrm{Cd}^{2+}$ to give $\mathrm{CdS}$ shell.

\subsection{Characterization of CdTe/CdS nanocrystals}

Ultraviolet-visible (UV-vis) absorption and fluorescence spectra were collected at room temperature with a SpectraMax M5 Multi-mode microplate reader (Molecular Devices) in the range of $200-800 \mathrm{~nm}$. The room temperature fluorescence quantum yield (QY) of nanowires was determined by comparing the integrated emissions of the nanowire solution with that of rhodamine $6 \mathrm{G}$ in ethanol $(\mathrm{QY}=95 \%)$ with identical optical density at the excitation wavelength. X-ray diffraction (XRD) measurements were performed on a Miniflex X-ray diffractometer (Rigaku) using Co K $\alpha$ radiation $(\lambda=0.1789 \mathrm{~nm})$ at $40 \mathrm{keV}$, at a scanning rate of $10^{\circ} \mathrm{min}^{-1}$ from $10^{\circ}$ to $90^{\circ}$. Transmission electron microscopy (TEM) characterization was conducted using a JEOL JEM 2100 electron microscope operating at an acceleration voltage of $200 \mathrm{kV}$. The elemental composition of the nanowires was determined by energy-dispersive X-ray spectroscopy (EDS) during TEM measurements. X-ray photoelectron spectroscopy (XPS) measurements were performed on a Kratos Axis Ultra X-ray photoelectron spectrometer (Perkin-Elmer).

\subsection{Cell culture}


Human embryonic kidney cells (HEK 293 cells) were cultured in RPMI-1640 medium, supplemented with $10 \%$ heat-inactivated fetal bovine serum (FBS) at $37{ }^{\circ} \mathrm{C}$ in a humidified atmosphere with $5 \% \mathrm{CO}_{2}$.

\subsection{Cell viability assay}

HEK 293 cells were seeded into 96-well plates (200lL containing $2.5 \times 10^{4}$ cells in each well). After incubation for $24 \mathrm{~h}$, the CdTe/CdS nanowires, which were dissolved in $20 \mu \mathrm{L}$ phosphate buffered saline (PBS) at the final concentration of 5-500 $\mu \mathrm{g} / \mathrm{mL}$, were added to each well. The cell samples were then incubated for $4 \mathrm{~h}$ at $37{ }^{\circ} \mathrm{C}$ under a humidified atmosphere $\left(5 \% \mathrm{CO}_{2}\right)$ in the dark. Then, the medium containing the nanocrystals was replaced by fresh culture medium. The cells were further incubated for 16 h. Colorimetric 3-(4,5-dimethylthiazol-2-yl)-2,5-diphenyl tetrazolium bromide (MTT) assays were performed to assess the cell viability. Briefly, at the end of each incubation, $20 \mu \mathrm{L}$ stock MTT ( $5 \mathrm{mg} / \mathrm{mL}$ ) was added to each well, and cells were then incubated for $4 \mathrm{~h}$ at 37 ${ }^{\circ} \mathrm{C}$. The medium was $\mathrm{r}$

each well. The cells were then incubated for another $0.5 \mathrm{~h}$ at 37 Tuorescent microscopic images was measured at $400 \mathrm{~nm}$ using Inverted Fluorescence Microscope of Leica DMI600 B. Cell viability, defined as the relative absorbance on each sample compared to that of a blank control, was calculated and expressed as a percentage. Statistical analyses were performed using the GraphPad Prism 5 software. Statistical significance was set at $\mathrm{P}<0.05$. 


\section{Results and discussion}

\subsection{Synthesis of Cysteine-Capped Straight CdTe/CdS Core/Shell Nanowires}

In recent years, colloidal CdTe nanowires have been prepared by various wet-chemical methods (Protasenko et al. 2006; Grebinksi et al. 2004; Grebinski et al. 2004; Kuno et al. 2006). These methods generally require the use of organic solvents, high temperature or pressure, oxygen and water-free conditions, and toxic reagents, such as three octyl phosphine (TOP three) and trioctylphosphine oxide (TOPO), all of which increase operational difficulty and preparation costs (Wang et al. 2011; Li et al. 2008; Li et al. 2009). The method described here is an environmentally friendly aqueous approach to the synthesis of CdTe/CdS core/shell nanowires through the reaction of $\mathrm{CdCl}_{2}$ with NaHTe in the presence of cysteine at atmospheric pressure and relatively low temperature $\left(100{ }^{\circ} \mathrm{C}\right)$. The nanowires are decorated with amino and carboxyl functional groups and can be well dispersed in aqueous solution. This preparation method has the advantages of simple operation and good controllability of the nanowires. The resultant nanowires have a core-shell structure, in which CdTe core nanowires are coated with a shell of higher energy band gap material (i.e. CdS) to enhance the quantum yield (QY) and to protect them from oxidation (Singh et al. 2012).

3.2 Effects of Reaction Time on the Morphology of Cys-capped CdTe/CdS

\section{Nanocrystals}


In our study, the reaction time has a significant influence on the morphology of the nanocrystals. Scheme 1 shows the evolution of nanowires as the reaction time is extended. Figure 1 displays typical TEM images of samples collected at $10 \mathrm{~min}, 30$ min, and 140 min reaction time. It clearly shows that by increasing the reaction time from 10 min to140 min, samples gradually changed from spherical nanoparticles to nanorods, and eventually completely turned into nanowires.

Scheme 1. Schematic illustration of the growth process of the Cys-capped CdTe/CdS core-shell structured nanowires.

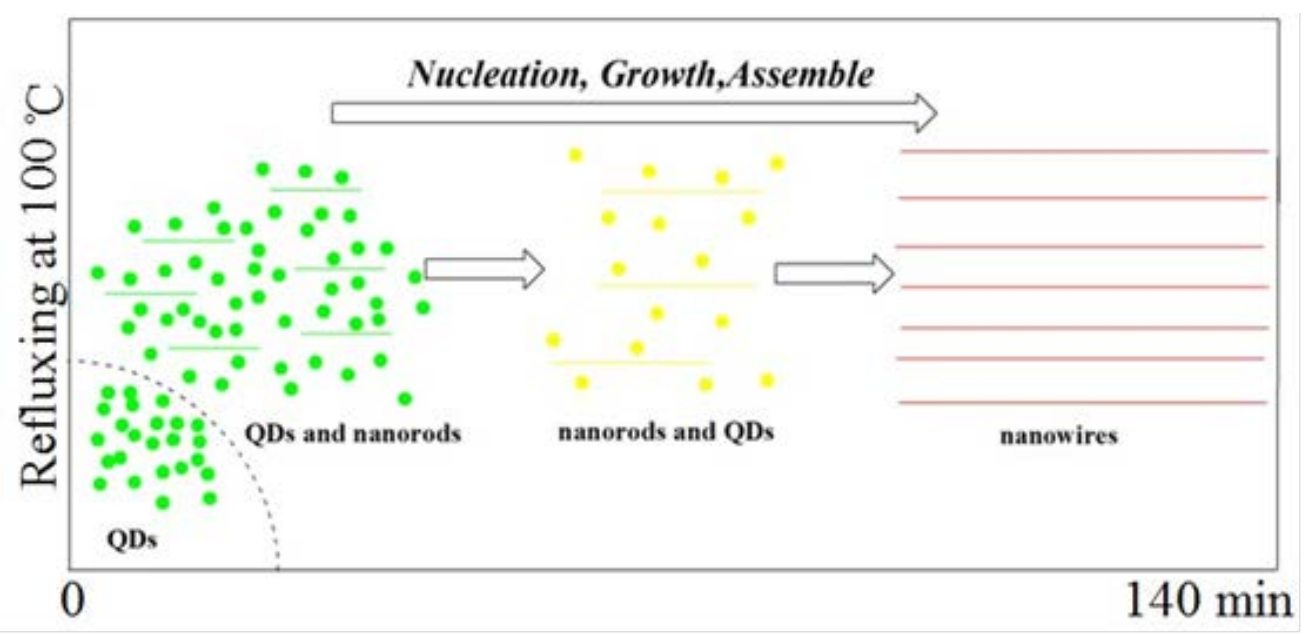

When the reaction time is short $(t=10 \mathrm{~min})$, the product mainly consists of quantum dots (QDs) and nanorods [Figure 1(a)]. The high-resolution TEM image of this sample in Figure 1(b) shows that some QDs are attached to the surface of the nanorods, suggesting that the nanorods are assembled from QDs and keep growing through Ostwald ripening (Wang et al. 2011). The particle size of the QDs is in the range of 2.5-3 nm, and the dimensions of the nanorods are 3-5 nm in diameter $(d)$ 
and 20-40 nm in length. Optical characterization demonstrates that CdTe/CdS nanocrystals at this stage have a fluorescence quantum yield of $87 \%$ at room temperature, comparable to the highest quantum yield of any nanocrystals prepared by other approaches.

As the reaction time was increased from $10 \mathrm{~min}$ to $30 \mathrm{~min}$, the diameter and length of the nanorods increased to $5 \mathrm{~nm}$ and $55-90 \mathrm{~nm}$ respectively, accompanied by a decrease in the amount of QDs in the sample. This result supports the occurrence of the Ostwald ripening and indicates faster growth in the length direction than in the diameter direction. Further prolonging the reaction time to 140 min leads to the formation of nanowires and the complete disappearance of QDs, we know that the wire can be shown with different shapes, like: curving or straight, from the Figure 1(e) we can see that the nanowires are almost straight. Compared with CdTe nanowires grown by the conventional VLS or SLS methods (diameter, $d=10.9 \pm 2.9 \mathrm{~nm}$ ) (Wang et al. 2011), the resulting nanowires are considerably thinner ( $d=\sim 5 \mathrm{~nm})$ with a narrower diameter distribution. They have a length in the range of 175 to $275 \mathrm{~nm}$, and their aspect ratio is between 1/35 and 1/55. The high resolution TEM image in Figure 1(f) clearly shows a planar spacing of $0.18 \mathrm{~nm}$, corresponding to the (111) planes of CdTe (Zhu et al. 2013). When the reaction time is prolonged to $180 \mathrm{~min}$, there is no change in the nanowires, which is also reflected by the similar UV-vis absorbance and photoluminescence spectra to those of nanowires collected at 140 min (Figure 2). Therefore, under the current preparation conditions, the growth of nanowires can be 
finished within $140 \mathrm{~min}$. Combine with the results we can know that the reaction time have more important to influence the morphology of the nanocrystal, so we can control the reaction time to obtain different morphology of the nanocrystal, like QDs, nanorods and naowires.

Figure 1. TEM and high-resolution TEM images of Cys-capped CdTe/CdS nanocrystals prepared in aqueous solution: (a-b) reaction $10 \mathrm{~min},(\mathrm{c}-\mathrm{d})$ reaction $30 \mathrm{~min}$, and (e-f) reaction $140 \mathrm{~min}(d=\sim 5$ $\mathrm{nm}$, length in the range of 175 to $275 \mathrm{~nm}$ ).
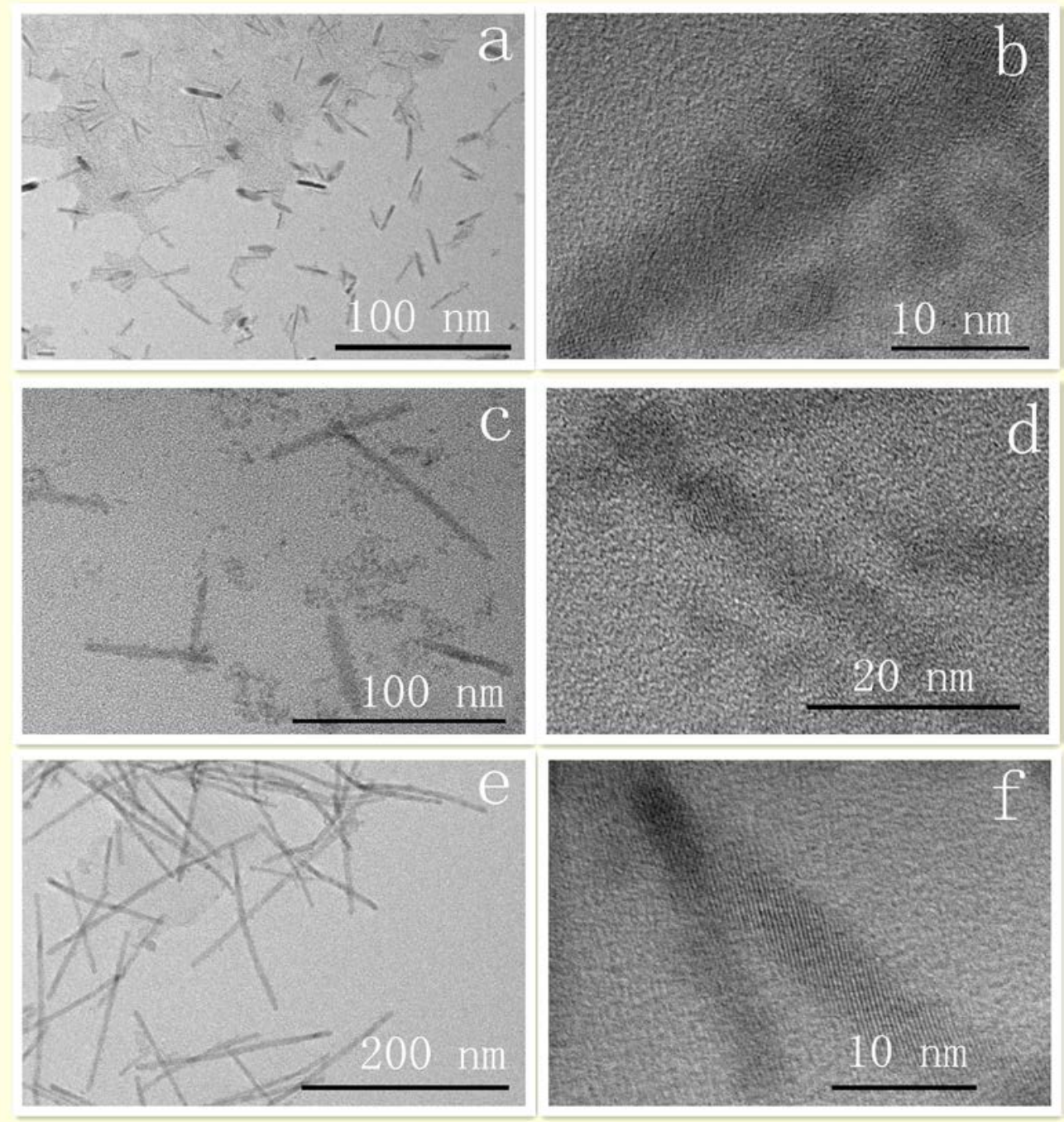


\subsection{Optical Properties of Cys-capped CdTe/CdS Nanocrystals}

Figure 2. Photograph of Cys-capped CdTe/CdS nanocrystal solutions under excitation of $365 \mathrm{~nm}$ UV lamp (top) and their corresponding UV-vis absorption (a) and PL spectra (b) after refluxing at $100^{\circ} \mathrm{C}$ for different times.
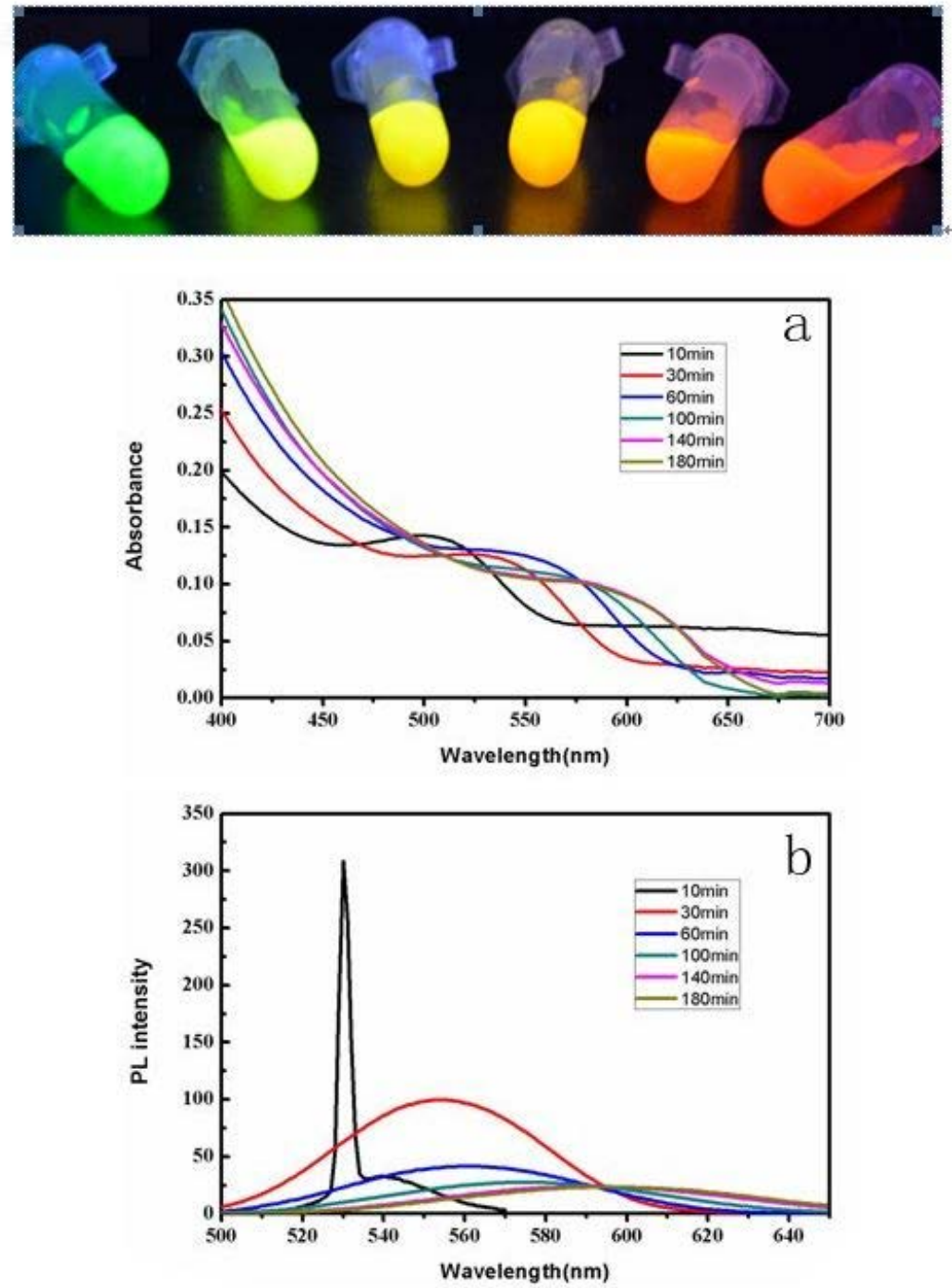
Figure 2 shows the evolution of the absorption and photoluminescence (PL) spectra of Cys-capped CdTe/CdS nanocrystals collected from the refluxing solution at 10, 30, 60, 100, 140, and $180 \mathrm{~min}$. The room temperature UV-vis absorption spectra showed a shift of the first exctionic absorption from $500 \mathrm{~nm}$ to $625 \mathrm{~nm}$, due to the increase in the nanocrystals' size as the refluxing time progressed from 10 to 180 min (Figure 2(a)). In addition, these nanocrystals show tuneable photoluminescence, ranging from green to yellow to red under an excitation of $360 \mathrm{~nm}$ UV light (Fig. 1(top)). Their emission peaks $\left(\lambda_{\text {em }}\right)$ correspondingly are shifted from $530 \mathrm{~nm}$ to $610 \mathrm{~nm}$ (Fig. 2(b)). Both UV-vis absorption and photoluminescence emission demonstrate the strong size dependence of the optical properties of the $\mathrm{CdTe} / \mathrm{CdS}$ nanocrystals. There is a narrow sharp peak at $530 \mathrm{~nm}$ in the emission spectrum of the sample collected at10 min (Figure 2(b)), which is attributed to the optimum-sized CdTe clusters. The broad peak centered at $540 \mathrm{~nm}$ arises from the large nanocrystals and nanorods. The fluorescence QY of this sample is as high as $87 \%$. With extended reaction time, the fluorescence intensity decreases rapidly, and the sample fluorescence QY is decreased to $29 \%$ when the reaction time is $30 \mathrm{~min}$. In the case of the final nanowires collected at 140 min, the fluorescence QY is decreased to 7.25\%. This value is much higher than for CdSe, CdTe, or the core-shell CdSe@CdS nanowires prepared by the SLS method (QY less than 1\%) (Wang et al. 2011; Li et al. 2012), which is due to the the presence of fewer defects in the core-shell structure and the good confinement of electrons and holes in the CdTe by the CdS shell. As mentioned previously, the optical properties of 
the sample prepared at $180 \mathrm{~min}$ are similar to those of the sample collected at 140 $\min$.

\subsection{Crystal Phase}

The crystal structure of the CdTe/CdS core-shell nanowires was investigated by high resolution TEM (HRTEM) and powder X-ray diffraction (XRD) techniques. Figure 3(b) shows XRD patterns of the CdTe/CdS core/shell nanowires synthesized via refluxing at $100^{\circ} \mathrm{C}$ for different times, i.e. $10 \mathrm{~min}, 30 \mathrm{~min}$, and $140 \mathrm{~min}$. The diffraction patterns indicate that all the nanowires belong to the cubic (zinc blende (ZB)) crystal structure. The broad diffraction peak is attributed to the smaller diameter of the CdTe/CdS nanowires $(d=\sim 5 \mathrm{~nm})$. Moreover, the diffraction peaks are all located between those of pure cubic CdTe （JCPDS 15-0770） and pure cubic CdS crystals (JCPDS 10-0454). At 10 min, the diffraction peaks are very close to those of CdTe crystals (Figure 3(b)), showing that the dominant structure at this point of time is cubic CdTe crystal phase. As the reaction time is prolonged, the diffraction pattern shifts towards larger $2 \theta$ angles, i.e. close to those of cubic CdS phase. Such a shift indicates the formation of a CdS shell on the nanowire surface (Zhu et al. 2013). Since the CdS shell can reduce surface defects of the core nanocrystals, it is believed that the CdS shell is essential for high fluorescence (Zeng et al. 2008). An HRTEM image of the ZB phase CdTe/CdS nanowires is shown in Figure 3(a). It demonstrates that the 
lattice fringes and the spacing correspond to ZB phase CdTe/CdS. It also shows that the nanowires grow along the [111] direction (marked by the arrow in Figure 3(a)). The EDS spectrum of the CdTe/CdS nanowires is shown in Figure 4. The elements S, $\mathrm{Cd}$, Te, and $\mathrm{Cu}$ are clearly observed, with the presence of $\mathrm{Cu}$ due to the copper grids used in EDS analysis. The presence of S, Cd, and Te in the individual nanowires supports the formation of core-shell structure of the CdTe/CdS nanowires, we know that the S element come frome the cystein. This indicate that we have successful used the cysteine to modify the CdTe/CdS nanowires' surface. From the Figure 4 we can see the the weight percent ratio of Cd:Te:S=1:5.3:27. However, we found that the proportion of Cd element is less than that of Te elements, which may be due to the nanowires is not very pure. Combined with XRD pattern (Fig. 3b) analysis found that there is an extra peak in (111) diffraction peak, which may be caused by excess Te.

Figure 3. a) TEM image of Cys-capped CdTe/CdS nanowire collected at $140 \mathrm{~min}$, b) typical XRD patterns of Cys-capped CdTe/CdS crystal grown at $100{ }^{\circ} \mathrm{C}$ for different times. 

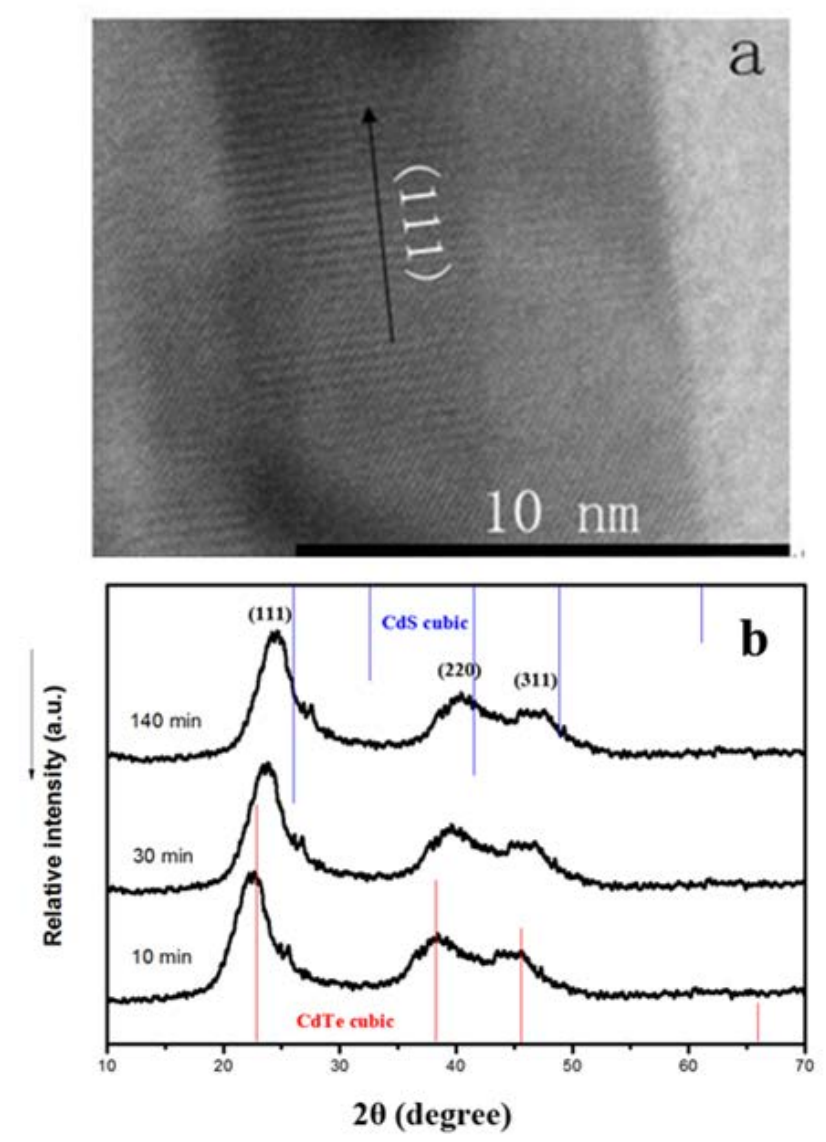

Figure 4. EDS spectrum of Cys-capped CdTe/CdS nanowires.

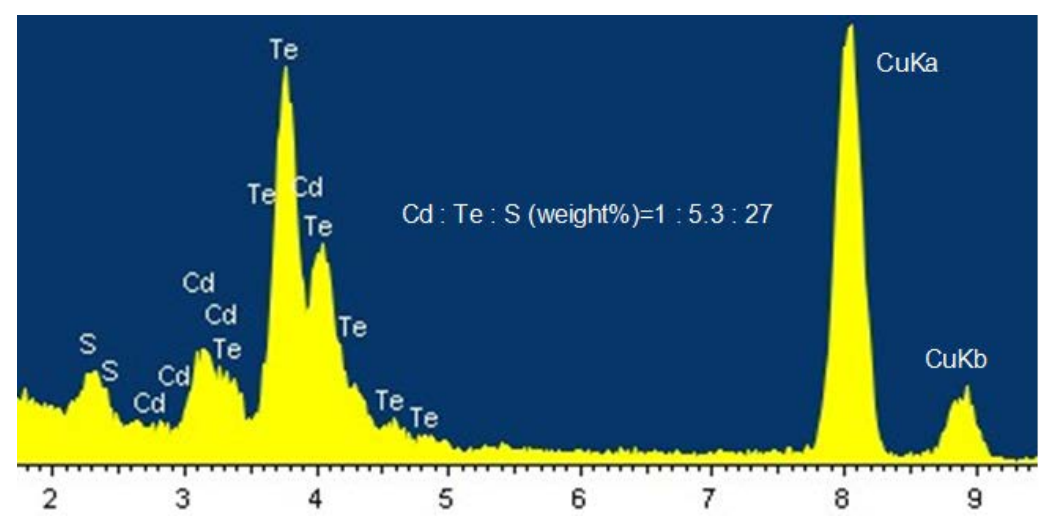

To further confirm the formation of the CdS shells, the samples were characterized with XPS. Their corresponding S2p spectra are shown in Fig 5. For the sample with the reaction time of $10 \mathrm{~min}$, the S2p spectrum can be fitted into five curves, including 
a doublet: S2p1/2 at 164.1 and S2p3/2 at $163.8 \mathrm{eV}$ (the blue lines). These binding energies are consistent with those for Cd-Sr complexes, demonstrating the formation of Cd-Cys complexes on the surface of the CdTe crystal (probably absorbed) and in solution (Bao et al 2004) .There is also a doublet attributed to S2p1/2 and S2p3/2 of CdS (Zhao et al. 2009), located at $163.0 \mathrm{eV}$ and $161.9 \mathrm{eV}$, respectively(the red lines). The other peaks at $165.0 \mathrm{eV}$ could be ascribed to S2p of disulfides. As the reaction time progressed, the integrated area of S from the CdS increased, while the area from the Cd-Cys complex decreased. This change indicates that the refluxing treatment leads to the formation of a CdS shell due to the decomposition of Cd-Cys at high temperature. In Figure 5 we can also see the high resolution TEM image of CdTe/CdS nanowires, we can observe that the diameter of nanowire's core at $\sim 2.4 \mathrm{~nm}$, the diameter of nanowire’s shell at $\sim 1.3 \mathrm{~nm}$.

Figure 5. XPS spectra of S2p recorded from Cys-capped CdTe/CdS crystal grown at $100{ }^{\circ} \mathrm{C}$ for different times. In all fitted spectra, solid and dotted lines represent $\mathrm{S}$ from CdS, Cd-Cys complexes, and disulfides, respectively. 


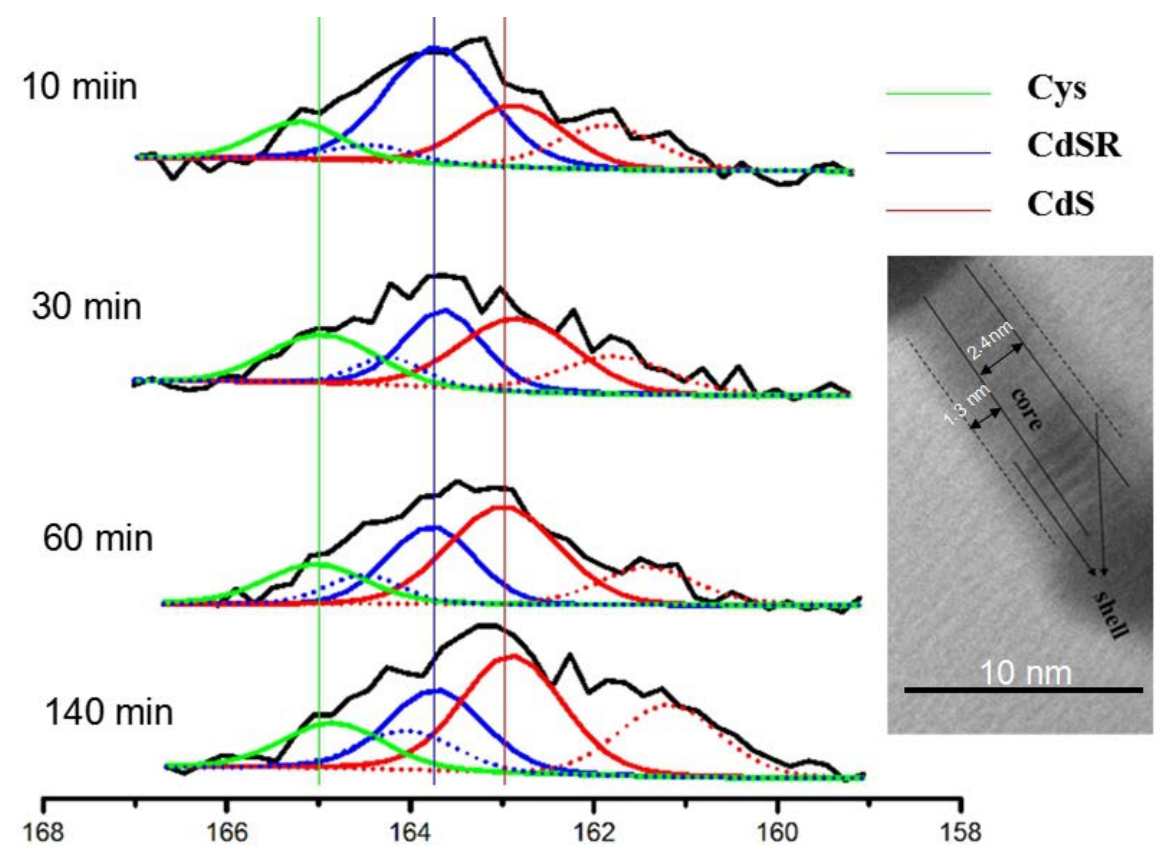

B.E. (eV)

\subsection{Cell Viability}

The bright photoluminescence and high stability of our Cys-capped CdTe/CdS crystals makes them very useful in bioapplications. We therefore assessed their cytotoxicity by the MTT assay. Different morphologies of Cys-capped CdTe/CdS nanocrystals were investigated (QDs obtained at $10 \mathrm{~min}$, nanorods prepared at $30 \mathrm{~min}$, nanowires obtained at $140 \mathrm{~min}$ ). Figure 6(A) shows that at the same concentration (50 $\mu \mathrm{g} / \mathrm{mL}$ ), the cytotoxicity of the three type of nanocrystals is in the order of QDs > nanorods $>$ nanowires, i.e. nanowires have the lowest toxicity, and cell viability is up to $78 \%$ after incubation for $16 \mathrm{~h}$. These results reveal that larger size CdTe/CdS nanocrystals are less cytotoxic than the smaller ones. The lower cytotoxicity of the larger nanowires might be ascribed to the protective effect of the CdS shell on the core CdTe (Zhu et al. 2013). It has previously been shown that the chemical bonds 
between $\mathrm{Cd}^{2+}$ and $\mathrm{S}^{2-}$ ions are more stable than those between $\mathrm{Cd}^{2+}$ and $\mathrm{Te}^{2-}$ ions under ambient conditions, which slows down the release of $\mathrm{Cd}^{2+}$ ions, leading to lower cytotoxicity of CdTe/CdS nanocrystals compared to bare CdTe QDs (Su et al. 2009). We also investigated the dependence of cell viability on nanowire concentration (Figure 6(B)). When the concentration was increased to $500 \mu \mathrm{g} / \mathrm{mL}$, only $20 \%$ of the cells were alive after $16 \mathrm{~h}$ incubation. In contrast, when the concentration was decreased to $5 \mu \mathrm{g} / \mathrm{mL}, \sim 90 \%$ of the cells survived after $16 \mathrm{~h}$ incubation.

Figure 6. Effects of Cys-capped CdTe/CdS crystals on cell viability in HEK 293 cell lines: (A) crystals synthesized with different reaction times $(10,30,140 \mathrm{~min})$ but the same concentration (50 $\mu \mathrm{g} / \mathrm{mL}$ ), and (B) same reaction time (140 min) but different concentrations (500 $\mu \mathrm{g} / \mathrm{mL}, 50 \mu \mathrm{g} / \mathrm{mL}$, $5 \mu \mathrm{g} / \mathrm{mL}$ ). Cells were treated with crystal solution for $4 \mathrm{~h}$, followed by incubation in fresh medium for $16 \mathrm{~h}$.

A

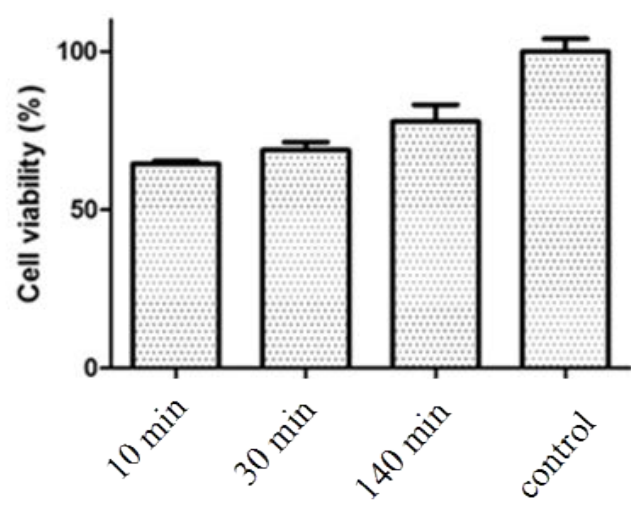

B

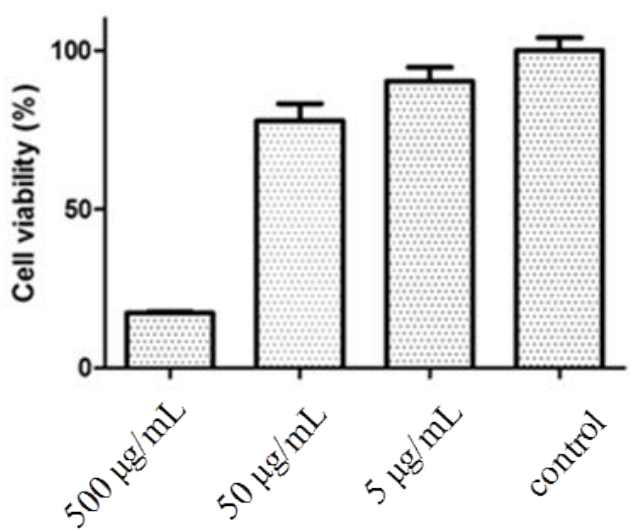

Figure 7. Fluorescent microscopic images of HEK 293 cells, incubated with Cys-capped CdTe/CdS 
nanowires synthesized with the same reaction time (140 $\mathrm{min})$ but different concentrations: $(A)$ control, (B) $50 \mu \mathrm{g} / \mathrm{mL}$, (C) $500 \mu \mathrm{g} / \mathrm{mL}$.
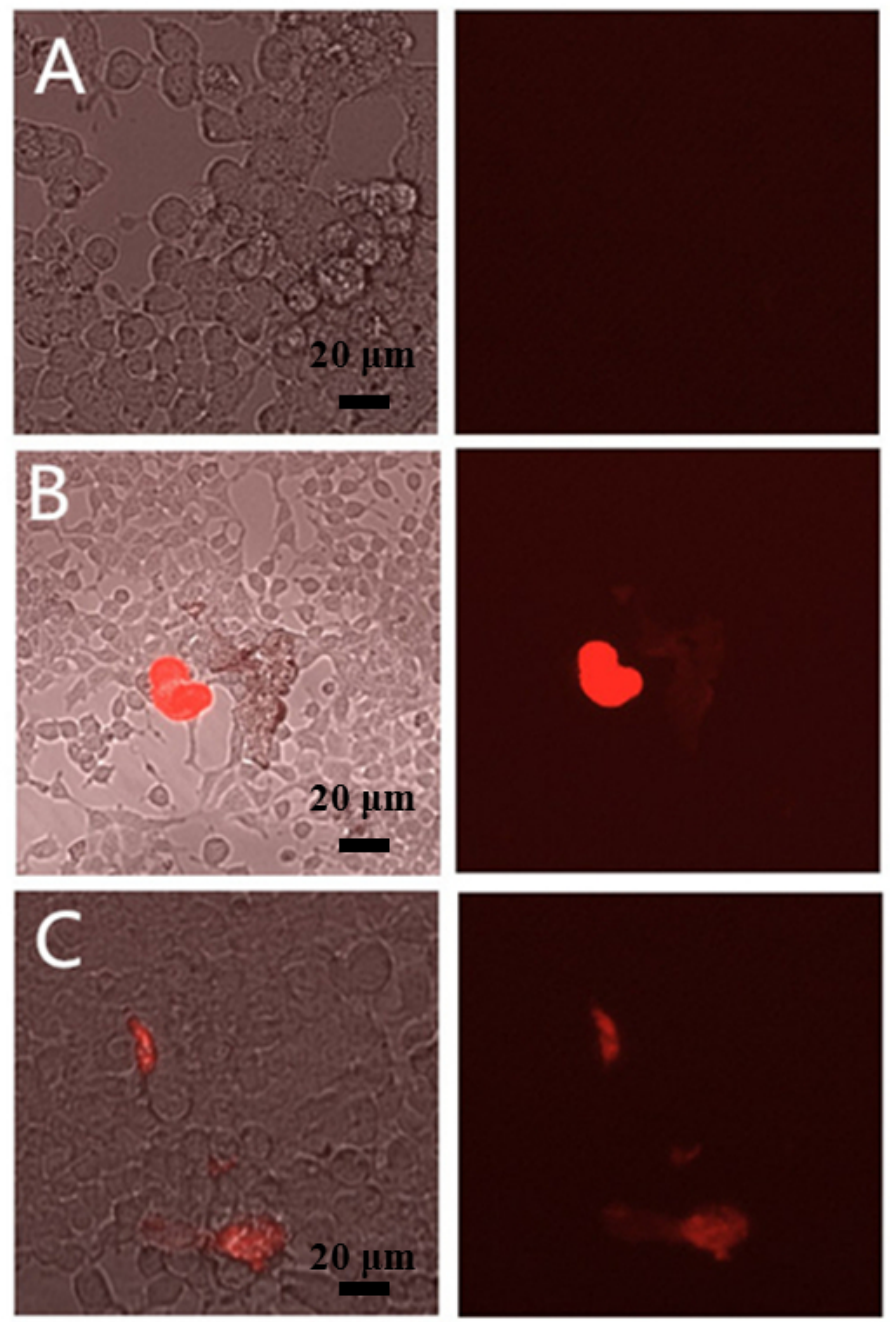

Fig. 7 shows fluorescence images of HEK 293 cells treated with CdTe/CdS nanowires at two concentrations. By comparing the morphology of the cells treated with PBS and CdTe/CdS nanowires, we conclude that CdTe/CdS nanowires had no significant effect on the cell growth at concentrations up to $500 \mu \mathrm{g} / \mathrm{mL}$ (Zhu et al. 2013). It's indicated that the Cys-CdTe/CdS nanowires have good biocompatibility, which is due 
to the nanowires have the core-shell structure, and functionalized with cysteine, which is a specific thiol-containing amino acid and serves an important structural role in many proteins. These results suggest that Cys-CdTe/CdS nanowires can be used to label and track cells in vitro, and their biocompatibility could be improved after coating with a biocompatible shell (Zhu et al. 2013).

\section{Conclusions}

In summary, a novel and simple route was developed to prepare CdTe/CdS core-shell nanowires in aqueous solution at atmospheric pressure. Highly fluorescent cysteine-capped straight CdTe/CdS core-shell nanowires were successfully synthesized by reacting $\mathrm{CdCl}_{2}$ with $\mathrm{NaHTe}$ in aqueous solution under refluxing at $100{ }^{\circ} \mathrm{C}$ for $140 \mathrm{~min}$. On increasing the reaction time from 10-140 min., CdTe/CdS nanocrystals gradually changed from QDs to nanorods, and eventually completely evolved into nanowires. The nanowires have amino and carboxyl functional groups and can be well dispersed in aqueous phase. The as-prepared CdTe/CdS nanowires show high fluorescence QY (up to 7.25\%) due to the unique properties of Cys and the formation of a CdS shell on the surface of the CdTe core. These nanowires are $5 \mathrm{~nm}$ in diameter and have a length of $175-275 \mathrm{~nm}$. The aspect ratio is in the range of 1/35 to $1 / 55$. The resultant nanowires have low toxicity and show potential in bioimaging, as indicated by using HEK 293 cells as an example.

\section{ACKNOWLEDGMENTS}


This work was supported by the National Natural Science Foundation of China (31160198); the High-End Technology Professionals Introduction Plan in Yunnan province (2010CI119); the Natural Science Foundation of Yunnan (2010FXW004); the Program for Innovative Research Teams (in Science and Technology) in the University of Yunnan Province (2010UY08, 2011UYN09); and the Yunnan Provincial Innovation Team (2011HC008). The authors wish to express their sincere gratitude for their financial assistance.

\section{REFERENCES}


Anton M, Christian S, Wang Z, Li Z, Tobias K, Alf M (2011) Diameter Scaling of the Optical Band Gap in Individual CdSe Nanowires. ACS Nano 5:7920-7927

Bao HB, Gong Y.J, Li Z, Gao MY (2004) Enhancement Effect of Illumination on the Photoluminescence of Water-Soluble CdTe Nanocrystals: Toward Highly Fluorescent CdTe/CdS Core-Shell Structure Chem Mater 16:3853

Bao HF, Wang EK, Dong SJ (2006) One-Pot Synthesis of CdTe Nanocrystals and Shape Control of Luminescent CdTe-Cystine Nanocomposites. Small 2:476-480 Carey CR, Yu YH, Kuno M, Hartlang GH (2009) Ultrafast Transient Absorption Measurements of Charge Carrier Dynamics in Single II-V Nanowires. J Phys Chem C 113: 19077-19081

Chen N, He Y, Su YY, Li XM, Huang Q, Wang HF, Zhang XZ, Tai RZ, Fan CH (2012) The cytotoxicity of cadmium-based quantum dots. Biomaterials 33:1238-1244

Consonni V, Rey G, Bonaimé J, Karst N, Doisneau B, Roussel H, Bellte D (2013) Synthesis and physical of Zno/CdTe core shell nanowires grown by low cost deposition methods. Appl Phys Lett 98:111906

Dagtepe P, Chikan V (2008) Effect of Cd/Te Ratio on the Formation of CdTe Magic-Sized Quantum Dots during Aggregation. J Phys Chem A 112:9304-9311 Deng DW, Qu LZ, Li Y, Gu YQ (2013) Versatile Self-Assembly of Water-Soluble Thiol-Capped CdTe Quantum Dots: External Destabilization and Internal Stability of Colloidal QDs. Langmuir 29:10907-1091 
Fernando P, Brian PT, Zheng GF (2007) Charles M. L. Nanowire-Based Nanoelectroni Devices in the Life Sciences. Mrs Bulletin 32:142

Goebl JA, Black R W, Puthussery J, Giblin J, Kosel TH. Kuno M (2008)

Solution-Based II-VI Core/Shell Nanowire Heterostructures. J Am Chem Soc $130: 14822$

Grebinksi JW, Richter KL, Zhang J, Kosel TH, Kuno M (2004) Synthesis and Characterization of $\mathrm{Au} / \mathrm{Bi}$ Core/Shell Nanocrystals: A Precursor toward II-VI Nanowires. J Phys Chem B 108:9745-9751.

Grebinski JW, Hull KL, Zhang J, Kosel TH, Kuno M (2004) Solution-Based Straight and branced CdSe Nanowires. Chem Mater 16:5260-5272

Gui RJ, An XQ (2013) Layer-by-layer aqueous synthesis, characterization and fluorescence properties of type-II CdTe/CdS core/shell quantum dots with near-infrared emission. RSC Adv 3:20959-20969

Hou JW, Yang XC, Lv XY, Peng DF, Huang M, Wang QY (2011) One-step synthesis of CdTe branched nanowires and nanorod arrays. Appl Surf Sci 257:7684-7688

Hwa SY, Kang SC, Pan KB, Kyung NK, Ho GJ, Kim YP, Chang HK (2009) Preparation and Characterization of Various surface-modified Semiconductor Nanocrystals. Korean Chem Soc 30:3137

Jay HC, Chee RE, Ajay A, Wong SM, Zhang GJ (2009) Label-Free Electrical Detection of Cardiac Biomarker with Complementary Metal-Oxide Semiconductor-Compatible Silicon Nanowire Sensor Arrays: Anal Chem 81:6266-6271 
Jiang F, Liu JJ, Li YC, Fan LZ, Ding YQ, Li YF (2012) Ultralong CdTe Nanowires:

Catalyst-Free Synthesis and High-Yield Transformation intp Core-Shell

Heterostructures. Adv Funct Mater 22:2402-2411.

Jiang F, Muscat AJ (2013) Solvent-Triggered Self-Assembly of CdTe Quantum Dots into Flat Ribbons. J Phys Chem C 117:22069-22078

Jiang F, Liu JJ, Li YC, Fan LZ, Ding YQ, Li YF (2012) Ultralong CdTe Nanowires:

Catalyst-Free Synthesis and High-Yield Transformation into Core-Shell

Heterostructures. Adv Funct Mater 22:2402-2411

Jiang XX, Chen F, Qiu WM, Yan QX, Nan YX (2010) Effects of molecular interface modification in CdS/polymer hybrid bulk heterojunction solar cells. Solar Energy Materials \& Solar Cells 94:2223-2229.

Kuno M, Ahmad O, Protasenko V, Bacinello D, Kosel TH (2006) Solution-Based Straight and Branched CdTe Nanowires. Chem Mater 18:5722-5732

Laura AB, Daniel HR, Gerald JM (2009) Selective Functionalization of Two-Component Magnetic Nanowires. Langmuir 19:7043-7048

Lee SE, Sasaki DY, Thomas DP, Daniel Y, Kamlesh DP, Lee PL (2009) Biologically Functional Cationic Phospholipid -Gold Nanoplasmonic Carriers of RNA. J Am Chem Soc 131:14066-14074

Liang HW, Liu S, Wu QS, Yu SH (2009) An Efficient Templa ting Approa ch for Synthe sis of Highly Uniform CdTe and PbTe Nanowires. Inorg Chem 48:4927-4933 
Li Z, Cheng L, Sun Q, Zhu Z H, Mark JR, Lu GQ (2010) Diluted Magnetic Semiconductor Nanowires Prepared by the Solution-Liquid-Solid Method. Angew Chem Int Ed 49:2777 -2781

Li Z, Ai JD, Sun Q, Muhsen A, Zhu ZH, Lu GQ (2012) Field-effect transistors fabricated from diluted magnetic semiconductor colloidal nanowires. Nanoscale $4: 1263$

Li Z, Ai JD, Sun Q, Muhsen A, Cheng LN, Mark JR, Zhu ZH, Cheng ZXm Wang XL, Jeremy H, Elmars K, Qiao SZ, Sean CS, Lu GQ (2011) Cobalt-doped cadmium selenide colloidal nanowires. Chem. Commun 47:1 1894-11896

Li Z, Andreas K, Anton M, Alf M (2008) Formation and Function of Bismuth Nanocatalysts for the Solution-Liquid-Solid Synthesis of CdSe Nanowires. Small 4:1698-1702

Li Z, Kurtulus ÖNF, Wang H, Andreas K, Ullrich P, Alf M (2009) Controlled Synthesis of CdSe Nanowires by Solution-Liquid-Solid Method. Adv Funct Mater 19:3650-3661

Luo BW, Deng Y, Wang Y, Tan M, Cao LL, Zhu W (2012) Fabrication and growth mechanism of zinc blende and wurtzite CdTe nanowire arrays with different photoelectric properties. CrystEngComm 14:7922-7928.

Newton JC, Ramasamy K, Mandal M, Joshi GK, Kumbhar A, Sardar R (2012) Low-Temperature Synthesis of Magic-Sized CdSe Nanoclusters: Influence of Ligands on Nanocluster Growth and Photophysical Properties. J Phys Chem C 
$116: 4380-4389$

Niu HJ, Zhang LW, Gao MY, Chen YM (2005) Amphiphilic ABC Triblock

Copolymer-Assisted Synthesis of Core/Shell Structured CdTe Nanowires.

Langmuir 21:4205-4210

Park WIl, Kim HS, Jang SY, Park J, Bae SY, Jung M, Lee H, Kim J (2008)

Transformation of ZnTe nanowires to CdTe nanowires through the formation of ZnCdTe-CdTe core-shell structure by vapor transport. J Mater Chem 18:875-880

Protasenko V, Bacinello D, Kuno M (2006) Experimental Determination of the

Absorption Cross-Section and Molar Extinction Coefficient of CdSe and CdTe Nanowires. J Phys Chem B 110:25322-25331

Rawalekar S, Kaniyankandy S, Verma S, Ghosh HN (2010) Ultrafast Charge Carrier Relaxation and Charge Transfer Dynamics of CdTe/CdS Core-Shell Quantum Dots as Studied by Femtosecond Transient Absorption Spectroscopy. J Phys Chem C 114:1460-1466

Simon KCL, Yu YG, Perez O, Puscas S, Thomas HK, Masaru K (2010)

Bismuth-Assisted CdSe and CdTe Nanowire Growth on Plastics. Chem Mater 22:77-8477

Singh N, Charan S, Sanjiv K, Huang SH, Hsiao YC, Kuo CW, Chien FC, Lee TC, Chen PL (2012) Synthesis of Tunable and Multifunctional Ni-Doped Near-Infrared QDs for Cancer Cell Targeting and Cellular Sorting. 
Bioconjugate Chem 23:421-430

Su YY, He Y, Lu HT, Sai LM, Li QN, Li WX, Wang LH, Shen PP, Huang Q, Fan CH (2009) The cytotoxicity of cadmium based, aqueous phase - Synthesized, quantum dots and its modulation by surface coating. Biomaterials 30:19

Tang ZY, Nicholas AK, Michael G (2002)Spontaneous Organization of Single CdTe Nanoparticles into Luminescent Nanowires. Science 297:12

Tian BZ, Zheng XL, Kempa TJ, Fang Y, Yu NF, Yu GH, Huang JL, Lieber CM (2007) Coaxial silicon nanowires as solar cells and nanoelectronic power sources. Nature 449:885-889

Utama MIB, Peng ZP, Chen R, Peng B, Xu X, Dong YJ, Wong LM, Wang SJ, Sun HD, Xiong QH (2011) Vertically Aligned Cadmium Chalcogenide Nanowire Arrays on Muscovite Mica: A Demonstration of Epitaxial Growth Strategy. Nano Lett. 11:3051-3057

Wang HL, Upmanyu M, Ciobanu CV (2008) Morphology of Epitaxial Core-Shell Nanowires. Nano Lett 8:4305-4311

Wang X, Ozkan CS (2008) Multisegment Nanowire Sensors for the Detection of DNA Molecules. Nano Lett 8:398-404

Wang Z, Li Z, Andreas K, Ma XD, Anto M, AlfMews (2011) Solution-Liquid-Solid Synthesis of Semiconductor Nanowires Using Clusters as Single-Source Precursors. Small 7:2464-2468

Wu HY, Meng F, Li LS, Jin S, Zheng GF, (2012) Dislocation -Driven CdS and CdSe 
Nanowire Growth. ACS Nano 6:4461-4468

Yang G, Jun Y, Chun S, Kin D, Kin J (2013) Catalytic growth of CdTe nanowires by closed space sublimation method. Thin Solid Films 546:375 -378

Yong SM, Muralidharan PSH, Kim DK (2011) One-step hydrothermal synthesis of CdTe nanowires with amorphous carbon sheaths. Mater Lett 64:1551-1554

Yu YG, Prashant VK, Masaru K (2010) A CdSe Nanowire/Quantum Dot Hybrid Architecture for Improving Solar Cell Performance. Adv Funct Mater 20:1464-1472

Zeng QH, Kong XG, Sun YJ, Zhang YL, Tu LP, Zhao JL, Zhang H (2008) Synthesis and Optical Properties of Type II CdTe/CdS Core/Shell Quantum Dots in Aqueous Solution via Successive Ion Layer Adsorption and Reaction. J Phys Chem C 112:8587

Zhao D, He ZK, Chan WH, Choi MMF (2009) Synthesis and Characterization of High-Quality Water-Soluble Near-Infrared-Emitting CdTe/CdS Quantum Dots Capped by N-Acetyl-l-cysteine Via Hydrothermal Method. J Phys Chem C 113:1293

Zhu Y, Li Z, Chen M, Cooper HM, Lu GQ, Xu ZP (2013) One-pot preparation of highly fluorescent cadmium telluride/cadmium sulfide quantum dots under neutral-pH condition for biological applications, J Colloid Interf Sci 390:3-10 